\title{
Structures in Fire, Yesterday, Today and Tomorrow
}

\author{
JEAN-MARC FRANSSEN \\ Dpt "Mécanique des matériaux et Structures" \\ Univ. of Liege \\ Chemin des Chevreuils 1 (bât. B52) \\ 4000 Liège 1 - Belgium
}

\begin{abstract}
This paper presents the author's view on various methods used for the evaluation of the behaviour of structures submitted to fire, namely experimental testing, tabulated data, simple calculation methods and numerical simulation. The evolution during the last decades, and the pertinence of these methods in different situations are discussed. The challenges that scientists and researchers are facing now and the problems that will need be addressed in the future are presented.
\end{abstract}

KEYWORDS: structures, fire resistance, simple calculation models, numerical simulations

\section{INTRODUCTION}

Fire safety engineering is a multidisciplinary science, or art, of which the fire resistance of the structure is only one component.

In some parts of the world, fire resistance has historically been given an overwhelming importance, probably as a consequence of some spectacular accidents that had triggered the attention of the public and of the authorities. In Belgium, for example, a fire in a department store in Brussels took the life of 323 people in May 1967 and the poor behaviour of the structure played an important role in the very bad outcome. In an excessive reaction, the authorities got themselves convinced that the more fire resistance they would require, the higher the safety level.

In other parts of the world, all that was required for the fire resistance of the structure was to put the necessary amount of thermal protection around the elements in order to limit the temperature increase to a certain level, irrespective of the load level or mechanical boundary conditions. The behaviour of the structure as a whole was totally ignored.

The situation is progressively changing, at a pace that has recently accelerated. On one hand, advocates of fire resistance are realising that other aspects cannot be neglected and may even in fact play a more crucial role. Statistics are, for example, showing that many more lives are taken by inhalation of toxic smoke products than by structural failure. On the other hand, a series of spectacular accidents have shown that a failure of the structural system can have dramatic consequences as well. The collapse of the WTC 1, 2, and 7 buildings in New York in September 2001 is certainly the event that took the highest life toll. Other events like the loss of the Piper Alpha platform in the North Sea in July 1988 with 167 casualties and the more recent fire of the Torre Windsor tower in Madrid in February this year have demonstrated that, even with no single life loss -as was the case in Madrid - , a failure in the containment function of the structure can result in enormous financial loss. In their career, fire fighters still have to suffer occasionally from less spectacular accidents like the one that took the life of 7 firemen in Switzerland in December 2004.

FIRE SAFETY SCIENCE-PROCEEDINGS OF THE EIGHTH INTERNATIONAL SYMPOSIUM, pp. 21-35

COPYRIGHT @ 2005 INTERNATIONAL ASSOCIATION FOR FIRE SAFETY SCIENCE 
There are various different methods to evaluate the behaviour of a structure subjected to fire. These methods are continuously improved and developed, and what was true yesterday may be questioned today and might be out dated tomorrow. This paper summarises the views of the author about the main families of methods. It discusses how they originated, what is their status nowadays, and what the near future is likely to bring.

\section{EXPERIMENTAL TESTING}

Experimental testing has been the first method available to assess the fire performance of structural elements. There are several severe circumstantial disadvantages to this method: high cost, long delays, limited number of facilities. More fundamental disadvantages are: limited size of the tested element, impossibility to investigate the behaviour of complete structures, uncertainty on the mechanical boundary conditions, almost total impossibility to make parametric studies.

Tests on small scale specimens have been performed in order to investigate the behaviour of complete structures including load redistribution. Although such tests may yield valuable results especially for steel structures, the validity of the scaling is sometimes questionable for more complex materials in which thermal and mechanical phenomena are not governed by the same dimensionless numbers. It is, for example, hard to scale down the evaporation of moisture linked to the temperature distribution in concrete elements.

On the other hand, there are very rare opportunities when complete structures can be tested under fire, if only under local fires. The tests series performed at BRE laboratories in Cardington is the most renowned example. Other buildings have been instrumented and burnt in the past but the measurements were primarily aimed at temperature distribution and the structural behaviour was observed more than measured.

Because of these limitations, the main field of application of experimental testing is research, either for new materials or new building systems. It can hardly be envisaged to perform experimental tests for assessing the fire resistance of a particular construction project, except if exceptional in nature and/or in size.

Experimental testing will nevertheless and probably forever remain one of the key tool of structural fire resistance assessment. The integrity criteria in separating elements, for example, cannot be predicted reliably by any other means. Some hypotheses that must be made for simple or numerical models can find their justification only in experimental testing. For example, will the concrete chambers of a composite steel concrete column remain in place and work together with the steel profile or will they separate from each other? Or will the bond between steel reinforcement and concrete be maintained in a reinforced concrete beam?

\section{TABULATED DATA}

Once a series of experimental tests has been performed on a significant number of similar elements, it quite natural to summarise the results in the form of tables, simple best fit equations or in a graphical presentation. All these belong to the family called "tabulated data" because the most usual way of representing the results is in the form of tables giving the fire resistance time as a function of a limited number of parameters. The same result can be achieved by the systematic application of calculation methods, either simple methods or general methods. 
Tabulated data are used mainly for masonry, concrete and composite steel-concrete elements, much less for steel structures.

They are quite valuable at the stage of pre-design when quick answers have to be found at a stage when all information on the structure that will be built is perhaps not yet available.

The main limitation is the fact that they apply to simple members usually subjected to the standard fire.

It would be highly beneficial for the application of these tables that some simple interpolation software be developed and made widely available. Some tabulated data recently proposed in Eurocode 2 [1] are based on so many parameters that the practical application becomes quite tricky.

It is also highly desirable that the background that is behind each tabulated data be presented in a clear and comprehensive manner and, preferably published in a widely available peer reviewed publication. It is too often that some tables are being used that appear to have been there forever but no one is able to retrieve exactly the primary information at the base of the table. It is the opinion of the author that some tables have been proposed recently whereas no information has ever been presented that would allow one to verify the validity of the proposal.

\section{SIMPLE CALCULATION MODELS}

Simple calculation models are calculation methods that are based on simple equilibrium equations of structural mechanics or of the theory of heat transfer. Most often, the equations are the direct extrapolation of similar equations valid at room temperature in which the effects of the deterioration of mechanical properties caused by elevated temperatures have been introduced. Some particularities that are typical for the situation of high temperatures may be incorporated in the model when required.

Simple calculation models have been developed for virtually any building material. They are not, generally speaking, limited to the standard fire but can be applied with several types of fire.

The first main limitations is that several different methods have to be applied for each different material or structural element. A concrete beam, for example, requires a different method than a steel beam or a concrete column. The second limitation is that simple models can hardly take into account the complex behaviour of a complete structure as a whole. The indirect effects of action that result from a certain level of restraint opposed to thermal expansion in complex structures, for example, can hardly be taken into account by simple models.

On the condition that the models are clearly and indisputably validated, they should deserve to be more widely spread. Typical structural engineers are still too reluctant to use them, considering that the analysis of a structure in the fire situation is something really peculiar. Simple computer software that renders the utilisation of the simple calculation models easier would help to promote a wider application of these models. The web site presented as [2], for example, proposes a simple computer software program for the fire design of simple steel elements. Strictly speaking, this model is on the verge of being outdated because it is based on the ENV version of Eurocode 3 [3] whereas the EN 
version should be published soon. It can anyway be used as a simple tool for predimensioning because the physical principals on which it is based are still valid.

\section{ADVANCED CALCULATION MODELS}

Advanced calculation model are those models based on a discretisation of the structure into elementary parts, the behaviour of each being analysed simply and the whole structure being reconstructed in matrices equations. Because the dimensions of the matrices are several orders of magnitude greater than what a human being can reasonably handle, these models require the utilisation of numerical software and computers. They are implemented by techniques known as finite element method (FEM), finite difference method or, more rarely, boundary elements method.

Advanced models must be based on acknowledged principles of structural mechanics and of the theory of heat transfer. They can be applied for various materials, provided that the material properties are known, for different types of fire, and they have the ability to model the behaviour of very large and complex structures, including the indirect effects of action that occur in these structures.

\section{Different Types of Tools}

The objective when the first numerical models where established was to make faster and at a cheaper cost in the computer what was done previously in the furnace tests. It was at that time considered that the modelling of structures subjected to fire was a very peculiar problem, and a multitude of specific software were developed in order to model each one very specific and particular type of element subjected to fire such as, say, steel beams, or concrete walls, or concrete columns. One research centre has even published one model for circular concrete columns and another model for concrete columns with square sections. All these are proprietary programs, usually established by individual researchers, typically for the purpose of their Ph. D. They have all their own merits, but will never be able to allow the analysis of any other element than the one for which they have been made, and most of them have seen their development stopped after a while. Except for very specific research projects and in particular situations, the development of such specific software should normally not be undertaken anymore.

With a greater maturity of the discipline, other software programs have been developed specifically with the objective of modeling structures in fire, but were efforts have been made to have a wider and more general field of application. They normally offer a library of different material models and of different finite elements that can be combined to adapt to different situations and structures. They typically result from a succession of numerous different $\mathrm{Ph}$. D. theses and research projects performed in a university department that has SiF (Structures in Fire) as his main research activity. Most of them are still confined to the research center were they are developed, although there is a recent tendency for these tools to be used also by other research centers or by design offices that specialized in fire safety engineering. The software SAFIR developed at the university of Liege by the author belongs to this family of software [4].

Commercially available software programs are used more and more nowadays. They have not been developed with the objective of modeling structures in fire, but they offer numerous possibilities, have great pre and post processing capabilities and have normally received extensive attention for validation. The price may yet be a problem, although some education licenses are usually offered for research purposes, and it is not so 
straightforward to get familiar with all the possibilities that have to be utilized in order to perform a SiF modeling analysis. On the condition that the user is really familiar with particular software, it is possible to constrain, or squeeze, such software in such a way that it performs a perfectly sound analysis of a structure in fire. One of the problem is that any user who is familiar with the code will end up with some results and with nice drawings, but a solid education and a high level of experience are also required in structural mechanics, in thermal problems, in non linear modeling and finally in the behavior of structures in fire before any confidence can be put in the results. This is of course also the case for the two other families of software but, for these other two, it becomes evident because these tools are specifically designed for the SiF situation; as a consequence, they are normally used only by SiF experts.

\section{What Happened Yesterday?}

Steel members have been the first objects to be modeled numerically, one of the reason being that the approximation of a uniform temperature in the cross section makes the calculation easier. It is now standard practice to consider a non uniform temperature distribution on the cross section of members, which makes it possible to analyze reinforced concrete or composite steel-concrete sections.

Except for establishing and validating more simple calculation models, advanced models are not used very often anymore for the analysis of simple elements. 2D structures such as frames, continuous beams or truss girders have been the most commonly type of object analyzed in the 90's. If out of plane stability had to be checked, it was not uncommon to make a separate verification for each member with a simple calculation model, on the base of the effects of actions provided by the $2 \mathrm{D}$ analysis performed with the numerical model. These days are gone now and 3D analyses are preferably performed, even if the structure analyzed is a plane frame; out of plane instabilities are thus automatically activated and there is no need for separate verifications.

Early models were systematically limited to the analysis of linear members such as beams, columns, frames or trusses. These were represented by beam or bar finite elements. It is now more and more common to see numerical models made on the basis of shell finite elements. One application is for modeling concrete slabs subjected to fire. Another type of application is the detailed representation of steel elements in which local buckling is to be expected.

More and more models are presented nowadays that are based on the utilization in the same model of a combination of several types of finite elements. Modeling any significant part of the Cardington composite building that has been submitted to various fire experimental tests requires the utilization of beam finite elements for representing the steel skeleton as well as shell finite elements for representing the slabs. Composite steelconcrete beams based on cellular steel profiles with openings in the web are prone to local buckling of the web post; an appropriate representation is based on shell elements for the steel profile and beam elements for the shear studs and the concrete slab. It is also possible to combine several types of elements with different levels of refinement in the discretisation when large structures have to be analyzed; the part of the structure subjected to the fire is represented in details whereas the parts that are away from the fire and remain cold and elastic are represented by less complex elements [5]. 


\section{What's Happening Today?}

The collapse of the World Trade Center buildings 1, 2, and 7 has resulted in a significant amount of resources being allocated to the analysis of the structural behaviour in these incidents. Some analyses have relied on substructures of limited sizes, but some analyses have been made on models the size of which had never been seen before in the fire community. It is not certain that the analysis of very large models will become the norm in the future. It is more likely that analysing such huge structures will remain an exception, triggered only by exceptional events.

A lot of effort is currently undertaken in order to better understand the behaviour of connections between different members during a fire. The numerical techniques that allow incorporation of the behaviour of semi-rigid connections in steel or composite frames are already available. More experimental testing has to be done in order to gather information on the actual behaviour of these connections and to provide the input data for the numerical codes. Simple models that allow the prediction of the behaviour of the joints have also to be developed. Some efforts have already been accomplished and simple models based on the component method have been proposed.

One of the barriers that all software faced until recently was the difficulty to pursue a numerical analysis of a structure submitted to fire when local and temporary failures were encountered. This happens, for example, when a secondary member fails by buckling because of restraint to thermal expansion. It is quite likely that the failure of this element would not endanger the stability of the complete structure, but the numerical analysis is systematically stopped by this local failure and the fire resistance may be highly underestimated. It has been shown that an analysis that considers the dynamic aspect of the behaviour, i.e., including acceleration and inertia terms, can be a satisfactory answer to this problem [6]. Dynamic analyses are more and more becoming standard practice and are indeed a must for any structure with a significant level of complexity. More information on this technique and the benefits that it brings are given in the next section.

\section{What's Coming for Tomorrow?}

Scientists around the world are still working to develop the capabilities of numerical modelling and its ability to better represent reality. The topics mentioned hereafter have already seen some development and promising results but some efforts have still to be undertaken before well established and recognized procedures emerge.

Most of the analyses performed in order to determine the temperature distribution in structural elements are based on a simple time-temperature equation that describes the evolution of the temperature of the gas considered as uniform in the compartment. Somewhat more refined models provide a distribution of impinging heat fluxes that can be applied at the boundary of the structural elements, see [7] for example. If a numerical analysis has been made using the Computational Fluid Dynamic (CFD) technique in order to describe the thermal environment in the fire compartment, the amount of information contained in the results is huge and it is not straightforward to transmit this information to the F.E code. Interconnecting FE and CFD codes poses various and complex problems, if only because it forces experts in very different fields to communicate. The practical problems, such as finding a common structure of information, are not conceptual but deserve a lot of attention. At the conceptual level, the most critical decisions deal with the levels of interaction that are considered or neglected as an approximation. Transmission of the thermal environment calculated by the CFD to 
the FE code is clearly within reach. It is the opinion of the author that, on the other hand, calculating the fire resistance of non loading partition walls by a FE code in order to transmit to the CFD code the time when the fire is transmitted beyond the room of origin is by far a more challenging goal and, for the time being, one that can not be obtained in the foreseeable future. This is because integrity failure of partition walls is dominated, except in very simple cases, by some phenomena that can not be predicted numerically such as the behaviour of local details, the presence and the behaviour of joints between adjacent panels, the localisation of crack openings, etc.

Spalling of concrete, when it occurs, poses a serious threat to reinforced and prestressed concrete structures subjected to fire. The threat is present especially for new concretes such as high resistance and self compacting concretes. Establishing a model that describes exactly this phenomena is highly desirable because it would help understand what factors are playing a key role and how the phenomena could be mitigated. It is anyway doubtful that it will ever be feasible to predict in advance the likelihood of spalling of a new concrete applied in a particular structure only from numerical models. Indeed, even in the hypothesis that a perfect and complete numerical model of spalling ever be established, it would require such an extended battery of experimental testing in order to provide the numerous material properties (porosity, permeability, tensile strength, fracture energy, modulus of elasticity, thermal elongation, transient creep, thermal properties, etc, all as a function of the temperature) that it would probably be as fast and much cheaper to fabricate a full size specimen of the structure and test it in a furnace.

Whereas nominal fire curves used in classification fire tests are continuously increasing, the temperature in a real fire enters in a decreasing phase after a certain amount of time and so do the temperatures in structural elements. Analysing numerically the behaviour of structures subjected to real fires requires also considering this cooling down phase. Some failures have indeed been observed in tests and sometimes also in numerical models during the cooling phase. The algorithms established for a situation of increasing temperature are not systematically valid and may have to be modified if a cooling phase has to be considered. One of the most important and difficult points is to obtain material properties at elevated but decreasing temperatures.

Modelling moisture movements is still a challenging task. It may yet prove necessary to model this phenomenon, not only for prediction of spalling in concrete, but also when charring occurs, in wood products typically.

Major efforts are still required for understanding and modelling the behaviour of some materials at elevated temperatures. For example, very little knowledge exists at high temperature on shear strength on concrete or on mechanical properties of gypsum

\section{EXAMPLE - DYNAMIC ANALYSIS}

\section{Introduction}

As an example of recent developments in "What's Happening Today" which will have a major influence on "What's Coming Tomorrow," we will consider dynamic analysis of structural collapse in fire.

The step by step numerical analysis of structures submitted to fire is traditionally performed by a succession of subsequent static analyses of the structure taking into 
account the modifications of the temperature field in the structure from one time step to the other, see for example [9 to 15]. Figure 1 shows schematically the algorithm of the usual procedure for a simple structure with a behaviour that can be characterised by one displacement and one force at a single varying temperature.

The load is first applied at ambient temperature, noted T0 on the figure. If the load level in case of fire is sufficiently high to generate a non linear behaviour of the structure, the load may be applied in several increments. On Fig. 1, two successive time steps have been used in order to apply the load in two increments. For each step, one initial displacement is first found corresponding to the incremental load, and a further iteration is then performed in order to treat the out of balance force that appears because of the non linear behaviour. It can be seen on the Figure that the obtained solution is not exactly converged, but the remaining out of balance force can be taken into account in the subsequent step so that they don't accumulate.

When the applied load is equal to the design load for the fire case, it is usually kept constant and the temperature in the structure is increased incrementally. Figure 1 illustrates the out of balance force that occurs because of the degradation of the mechanical properties of the structure during the first temperature increase, from time step 2 to time step 3 . This out of balance force leads to a new incremental displacement.

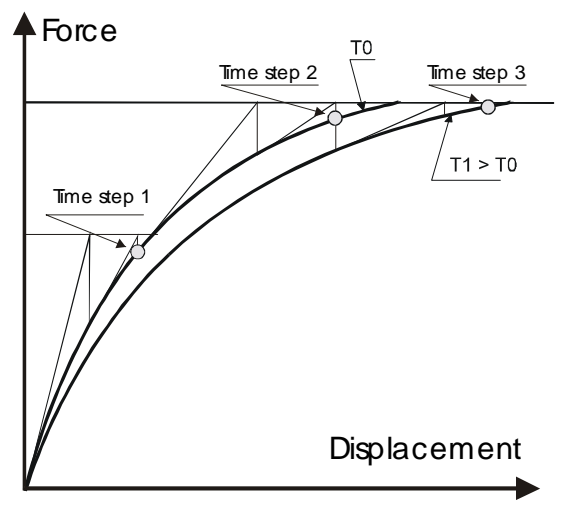

Fig. 1. Schematic algorithm for a static analysis.

If the loads at all degrees of freedom of the structure are noted $\{\boldsymbol{F}\}$ and if the corresponding displacements that have to be determined are noted $\{\boldsymbol{u}\}$, then Eq. 1 is used to determine the incremental displacements.

$\{\Delta F\}=[K]\{\Delta u\}$

where $[\boldsymbol{K}] \quad$ is the stiffness matrix of the structure, represented on Fig. 1 by the slope of the curve at each point.

$\{\Delta \boldsymbol{F}\}$ represents either the increment of external applied forces or the out of balance forces during the iteration process.

In a simple structure, the evolution of the situation toward failure during a fire is depicted by Fig. 2 where the last converged time step is time $\mathrm{N}^{\circ} 4$. For any higher temperature, the behaviour of the structure has degraded to such an extend that it is not possible to find a point on the curve 5 that satisfies the equilibrium. In other words, from point 4 , it is 
possible to increase the displacements of the structure without increasing of the load. The structure is unstable. Mathematically, this corresponds in Eq. 1 to the fact that the stiffness matrix is not anymore positive defined.

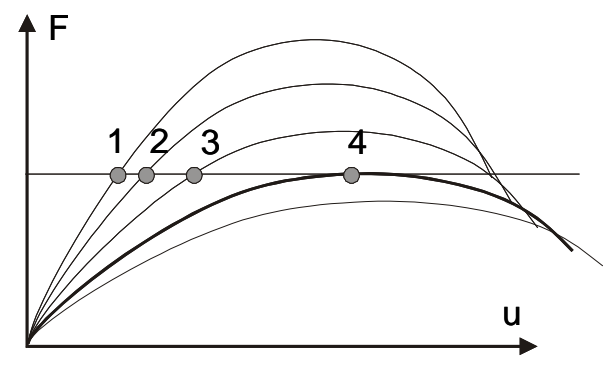

Fig. 2. Evolution toward failure in a simple structure.

For some more complex structures yet, the behaviour may be more complex. On Fig. 3 for example, point 3 seems to be the last possible converged point because the structure is unstable for any higher displacement (or for any higher temperature) whereas it can be observed that another point of equilibrium exists at temperature T4, but for a significantly larger displacement. Such a response of the structure can be generated either by material or by geometrical instabilities.

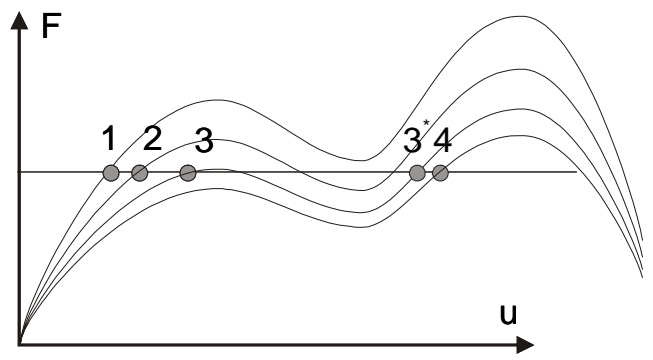

Fig. 3. Snap through.

In some cases, the instability affects the complete structures. This can be the case, for example, for a concrete beam on 3 supports when the negative bending moment on the central support leads to a severe cracking in the section. In this case, we would call it a temporary failure because, if the software is able to cope with this phenomena and finds another position of equilibrium corresponding to larger displacements of the beam, the simulation can be run for a longer duration after this instant of temporary instability.

In some other cases, the instability affects only one part of the structure. This can be the case, for example, for a member under compression in a statically indeterminate structure. The buckling of this single element may put the algorithm in jeopardy, whereas the rest of the structure might be able to survive for a longer duration, even if this element is completely removed. In this case, we would call it a local failure.

\section{Various Attempts to Solve the Problem}

Reducing the time step to very small values has no effect on the problem mentioned here because, even if it is approached by very small time steps, there is a moment when the structure is unstable and it appears that the software is totally unable to perform any simulation beyond that moment. On the contrary, it may happen that an artificially or 
inadvertently chosen larger time step would allow the software to "jump," for example, from point 2 to point 4 on Fig. 3. This is by no means a reliable solution as success is far from being guaranteed.

It is tempting to modify the material constitutive models in order to delete the unstable characteristics. The uniaxial relationship of steel proposed by Eurocode 3 [3], for example, has an horizontal plateau with no stiffness for a stress related strain extending from $2 \%$ to $15 \%$. A very small slope here can help bring some small stiffness in the structure that may help somehow. It is even more tempting to disregard the descending branch that is present in this steel model from $15 \%$ to $20 \%$ and to replace it by a infinitely long plateau. Apart from the fact that these solutions yield to the presentation of results that are falsely labelled as "Eurocode model," they are not general either because they are specific to some materials and by no means solve the cases when the instability is caused by geometric reasons.

It is possible to consider the true stress-strain relationship when evaluating the out of balance forces, but using a modified stiffness when calculating the stiffness matrix. For example, the descending branch of the concrete model [1] can be considered exactly when evaluating the stresses, but a zero stiffness is considered instead of the true negative value when evaluating the stiffness matrix. This is not incorrect because only the out of balance forces have to be evaluated exactly for the non linear process to converge. In fact, an approached value of the stiffness matrix is used leading to a modified NewtonRaphson convergence algorithm instead of the true Newton-Raphson algorithm that was implied on Figs. 1, 2, and 3. On these curves, the linear branch leaving any out of equilibrium point does not need to be exactly tangent to the curve at that point in order to reach convergence. Convergence with an approximated stiffness matrix may require a larger number of iterations. The same shortcomings exist here: success is not guaranteed, the solution is specific to some specific materials and geometrical instabilities are not addressed.

Another family of solutions are the so called Risk types or arc-length methods. The author has previously implemented such a method in his own code [4]. After a traditional procedure, when an unstable situation is approached, for example point 3 on Fig. 3, the temperature is kept constant and this method is activated to travel on the curve from point 3 to the other point of equilibrium, point $3^{*}$ on the Figure, this supposed to occur in the same time step. The principle of these methods is that the applied loads are multiplied by a scalar load multiplier, which constitutes a new unknown to the problem, and a new constraint equation is added, expressing the fact that each obtained point is at a given distance (the arc length) from the previous point in the hyper space that contains all the loads and displacements. This method has some merits such as the fact that it is not material dependent, does not require any modification of the recommended material models and can treat geometrical instabilities as well. Some successes have been obtained with this method [11] but time has shown that it can also fail in many cases so that it can not be considered as sufficiently general. Furthermore, it may not be without any consequences to unload then reload the total structure that has an inelastic response, when only one part of it is creating the problem. For example, if a diagonal at the upper floor of a complex building is entering into buckling, is it really innocuous to numerically unload then reload the heavily loaded concrete columns that may be present at the first floor? 


\section{The Dynamic Approach}

The idea is to model the behaviour of the structure as a dynamic process with the objective that acceleration term will counterbalance the negative stiffness during the unstable states of the structure. Equation 2 is the basic equation for a dynamic analysis if the behaviour of the structure is linear.

$$
\{F\}=[K]\{u\}+[C]\{\dot{u}\}+[M]\{\ddot{u}\}
$$

where $[C] \quad$ is the damping matrix,

$[M]$ is the mass matrix,

$\{\dot{u}\},\{\ddot{u}\}$

are the velocity and acceleration at the nodes, to be determined as well as the displacements.

In SAFIR, the Newmark method has been used for the time integration of Eq. 2 and the contribution to the mass matrix coming from the finite elements has been diagonalised in order to limit memory allocation requirements [6]. The shell finite element has only the terms corresponding to displacements in the mass matrix (no rotation). For the 3D beam finite element, the 2 masses in rotation around the axes that are perpendicular to the longitudinal axis are given the same value as the mass in rotation along the longitudinal axis. This approximation is justified by the fact that several finite elements are anyway used to model one beam member. It does not necessitate to rotate the mass matrix from the local to the global system of coordinates. Nodal masses can also be added by the user, either for displacement or for rotation type degrees of freedoms. A numerical damping has been introduced instead of the more traditional Rayleigh damping; the Newmark parameters are modified. In SAFIR they have been chosen as $\delta=0.80$ and $\alpha=0.45$. It has to be kept in mind that the aim is not the precise modelling of dynamic effects where a precise determination of the accelerations and of the damping is required as would be the case, for example, in a design of a building against seismic actions. An automatic adaptation of the time step during the analysis has been implemented, based on the number of iterations required for obtaining convergence. If convergence is not obtained, the software automatically comes back to the previously converged point and tries again with a reduced time step.

The required CPU time may be longer than for static analyses, but the time required for each time step is not significantly increased. A longer CPU time is mainly required when very small time steps have to be used.

\section{Examples and Case Studies}

The first example is academic but illustrates quite well the possibility to cope with the local failure, here the buckling of a diagonal, see Fig. 4. The steel frame made of HEA100 sections for the columns and IPE140 for the beam is subjected to a horizontal load (3000 N). The frame is able to withstand this load, but a pinned diagonal bracing (HEA100) has nevertheless been introduced in order to limit the horizontal displacement. 


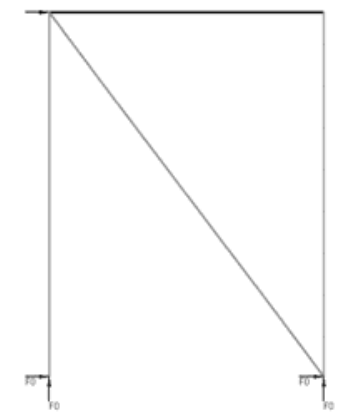

Fig. 4. Bucking of a diagonal.

The diagonal is the sole member to be heated by the ISO fire. It thus tends to elongate but the members of the frame prevent this movement. The compression in the diagonal increases while its mechanical properties decrease and buckling occurs after 3300 seconds. The horizontal displacement at the top of the diagonal first exhibits a movement to the left due to thermal elongation, a progressive stabilisation, then a sudden buckling to the right with some oscillations, and a final stabilisation for a displacement to the right equal to $103 \mathrm{~mm}$ (exactly the value that can be calculated for the frame without any bracing), a situation that the remaining structure can survive indefinitely, whatever the temperature of the diagonal.

Figure 5 shows the increase of axial load in the diagonal until buckling and, after buckling, the decrease toward a nearly zero value of this force. The structure truly lives on as if the buckled diagonal was not there. A traditional static algorithm is unable to cope with this local buckling and the analysis stops irremediably at 3500 seconds.

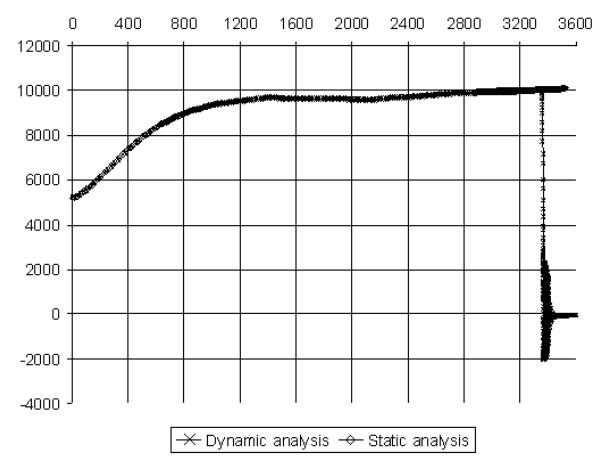

Fig. 5. Axial force in the diagonal.

Figure 6 shows the deflected shape at failure of a series of steel portal frame, submitted to a local fire. Failure time with the static analysis is 769 seconds, caused by buckling in one purlin. A dynamic analysis, on the other hand, shows that the heated frame is stable until 1257 seconds although, in this case, failure of the heated frame seems to produce failure of some of the parallel frames. 


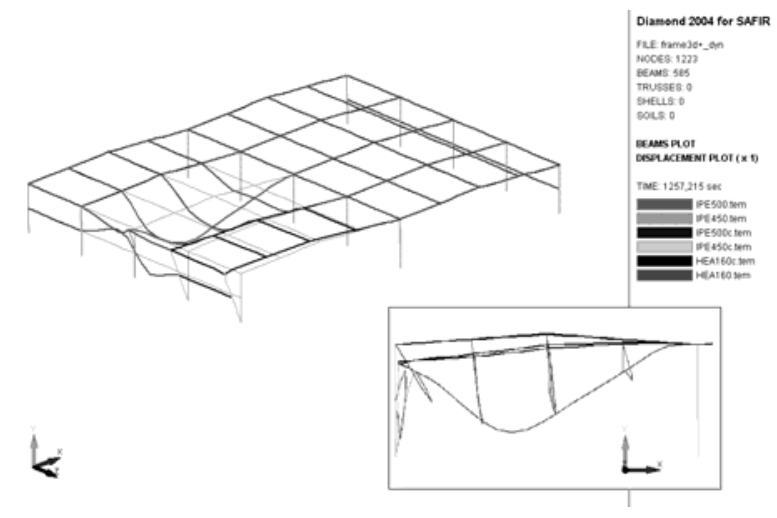

Fig. 6. One frame and some purlins heated.

Figure 7 shows the deflected shape at failure (amplified by a factor of 20) of an 87 by $28 \mathrm{~m}^{2}$ reinforced concrete flat slab supported at concentrated points. This slab is analysed at room temperature in order to determine the ultimate load bearing capacity in bending. A static analysis could not cope with the high level of load redistribution when plasticity occurs, mainly in the regions near the supports.

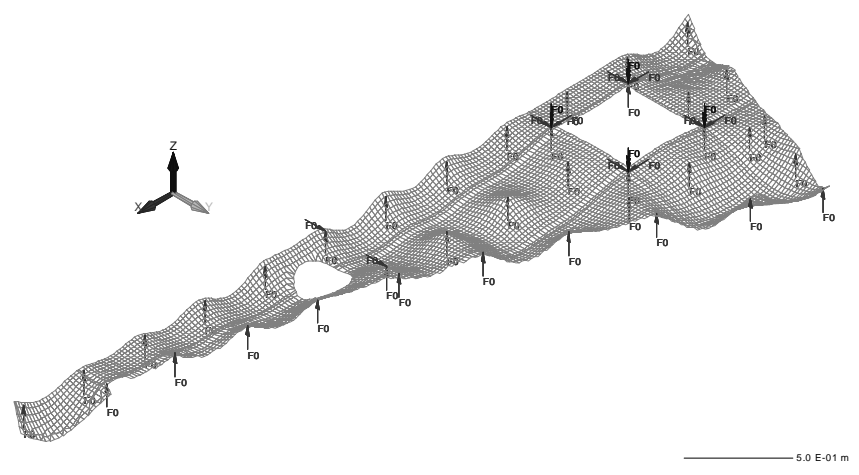

Fig. 7. Concrete slab at room temperature (courtesy Batiserf).

Figure 8 shows the deflected shape of a heated short cellular steel beam at failure. Whereas the static analysis stops at 856 seconds, the dynamic algorithm was able to run until 862 seconds. The failure time is here nearly the same, but the final displacements are much larger in the dynamic analysis. Indeed, in the static analysis, the final displacements of the structure are nearly invisible without an amplification factor. More amazing is also the fact that, whereas the geometry and the loading are nearly symmetric (the initial out of straightness of the beam is not), the dynamic algorithm was able to run until the moment when failure is clearly localised at one of the two supports. 


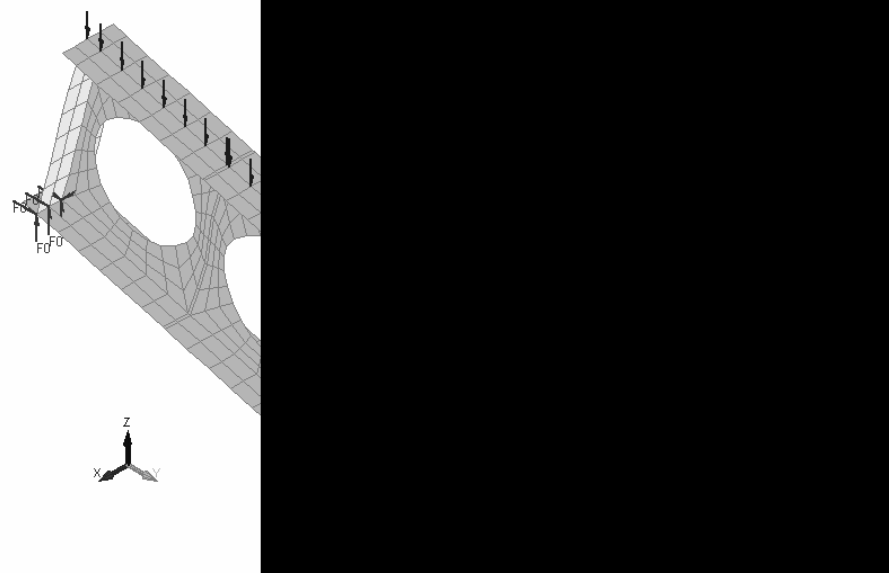

Fig. 8. Heated short cellular steel beam (courtesy ARBED).

\section{CONCLUSIONS}

Modelling the behaviour and estimating the fire resistance of structures subjected to fire is a discipline that has now reached a certain level of maturity.

Different methods with different levels of sophistication are available. Experimental testing will forever remain one the pillars of the discipline, either for gathering data on material properties or to observe complex behaviour in new structural elements. Tabulated data allows the determination of the sizes of structural elements very quickly and easily at a preliminary stage in the design of a structure. Simple calculation model allow checking the stability of structural elements on the base of equilibrium equations. Advanced numerical models are the preferred tools for modelling the behaviour of complex structures as realistically as possible.

All methods have their own limitations that have to be identified and recognised very clearly. Efforts are continuously undertaken in other to develop further these methods, to extend their capabilities and to make them more reliable. Most of the efforts nowadays are dedicated to the advanced numerical models.

\section{REFERENCES}

[1] Eurocode 2: Design of concrete structures - Part 1-2: General rules Structural fire design, prEn 1992-1-2, CEN, Brussels, March 2004.

[2] http://www.ulg.ac.be/matstruc/en/Download.html, download ELEFIR.ZIP.

[3] Eurocode 3 : Design of steel structures. Part 1.2 : General rules. Structural fire design, ENV 1993-1-2, CEN Brussels, 1995.

[4] Franssen, J.M., "SAFIR - A thermal/Structural Program Modelling Structures Under Fire,” Engng Journal, AISC, accepted for publication, 2005.

[5] Liew, J.Y.R., and Chen, H., "Direct Analysis for Performance-based Design of Steel and Composite Structures," Progress in Structural Engineering and Materials, John Wiley, UK, 6, No. 4, pp. 213-228, (2004). 
[6] Franssen, J.M., and Gens, F., "Dynamic Analysis Used to Cope with Partial and Temporary Failures," SiF'04, Structures in Fire, Proc. of the 3rd Intl Workshop, NRC-CNRC, IRC, Ottawa, Franssen, Benichou, Kodur \& Sultan ed., (2004), pp. 297-310.

[7] Eurocode 1: Actions on structures - Part 1-2: General actions - Actions on structures exposed to fire, EN 1991-1-2, CEN Brussels, Nov. 2002.

[8] Quast, U., Hass, R., and Rudolph, K., "STABA-F: Berechnung des Tag-und Verformungsverhaltens von einachsig gespannten Bauteilen unter Feuerangriff," T. U. Braunschweig, 1984.

[9] Franssen, J.M., "Etude du comportement au feu des structures mixtes acier béton,” Ph. D. thesis, Collection de la F.S.A., N¹11, Univ. de Liège, 1987.

[10] Zhao, B., "Modélisation numérique des poutres et portiques mixtes acier-béton avec glissements et grands déplacements : résistance à l'incendie, ” $\mathrm{Ph}$. D. thesis, INSA, Rennes, 1994.

[11] Kaneko, H., "Etude par la méthode des éléments finis du comportement mécanique d'éléments plaques en acier soumis à l'incendie." Construction Métallique, C.T.I.C.M., 1, pp. 37-50, (1990).

[12] Becker, J., Bizri, H., and Bresler, B., "Fires-R.C. A Computer Program for the Fire Response of Structures - Reinforced Concrete,” Report UCB FRG 74-3, Univ. of California, Berkeley, 1974.

[13] Huang, Z., Burgess, I.W., and Plank, R.J., "Fire Resistance of Composite Floors Subject to Compartment Fires," J. Construct. Steel Research., 60, Iss. 2, pp. 339-360, (2004).

[14] Forsen, N. E., "A Theoretical Study on the Fire Resistance of Concrete Structures,” The Norwegian Inst. of Technology, Trondheim, 1982.

[15] Anderberg Y., "Fire-exposed Hyperstatic Concrete Structures - An Experimental and Theoretical Study,” Lund Institute of Technology, 1976.

[16] Franssen, J.M., "Failure Temperature of a System Comprising a Restrained Column Submitted to Fire," Fire Safety Journal, 34, pp. 191-207, (2000). 
